\title{
Computational Models for New Fiber Optic Tweezers
}

\author{
R. S. RODRIGUES RIBEIRO ${ }^{1,2 *}$, P. A. S. JORGE ${ }^{1}$, and A. GUERREIRO ${ }^{1,2}$ \\ ${ }^{1}$ INESC-TEC (coordinated by INESC Porto), Rua do Campo Alegre, 687. 4169007 Porto, Portugal \\ ${ }^{2}$ Departamento de Física e Astronomia, Faculdade de Ciências, Universidade do Porto, Portugal \\ ${ }^{*}$ Corresponding author: R. S. RODRIGUES RIBEIRO $\quad$ E-mail: ritbeiro@gmail.com
}

\begin{abstract}
This paper discusses the calculation of the trapping forces in optical tweezers using a combination of the finite differences time domain (FDTD) method and the Lorentz force on electric dipoles. The results of 2D simulations of the trapping of a circular particle by a waveguide with a circular tip are presented and discussed.
\end{abstract}

Keywords: Optical tweezers, optical fiber, single cell, FDTD, electromagnetic force, biosensors

Citation: R. S. RODRIGUES RIBEIRO, P. A. S. JORGE, and A. GUERREIRO, "Computational Models for New Fiber Optic Tweezers," Photonic Sensors, vol. 3, no. 1, pp. 57-60, 2013.

\section{Introduction}

This paper presents a simulation method for computing the force generated by an electromagnetic field produced by a steering and focusing waveguide on small dielectric particles. This method combines the calculation of the stationary state of the electromagnetic field in a system composed of a waveguide and a dielectric particle, as shown in Fig. 1, using the electromagnetic equation propagation (MEEP) implementation of the finite differences time domain (FDTD) method, developed at the Massachusetts Institute of Technology (MIT), with the Lorentz equation for the force on an electric dipole. This method can be used to study and optimize trapping in optical tweezers (OT), for example, for applications in single cell manipulation in suspension or in optofluidic platforms [1].

With the advent of microfluidic and optofluidic technologies [2, 3] it has become possible to manipulate single cells in a contactless minimally invasive fashion. This can be used to facilitate single cell biosensing, for example, single cell genomics, proteomics, and metabolomics [4].

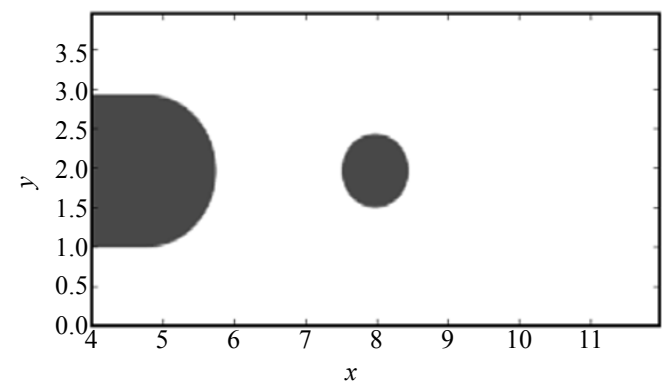

Fig. 1 Simulation model is composed of a waveguide structure (in grey on the left) and a circular dielectric particle (in grey on the right), both with a relative dielectric constant of 2.88 : a point source particle is located along the optical axis of the waveguide at $x=1.1$, and the spatial units are in micrometers.

Most advanced OT systems rely on bulk elements comprising a trapping laser, beam expansion and steering optics, high numerical aperture (NA) objective, holder, and elements that allow dynamic control over the position and stiffness of the trapping force: typically acousto-optic deflectors or liquid crystals space light modulators (SLM) are used. Such setups are often

Received: 17 July 2012 / Revised version: 29 July 2012

(C) The Author(s) 2012. This article is published with open access at Springerlink.com 
incorporated in an inverted microscope adding the imaging capability. While these approaches enable advanced features such as multiple point holographic trapping and time multiplexing of trapping points, their implementation is often bulky and expensive, thus preventing their widespread use [5]. Furthermore, operation of bulk OT setups in turbid media or thick samples is often compromised by difficulties in focusing. This hinders the use of this technique in unprocessed samples or in vivo applications $[1,5]$.

In this context, fiber optics based tweezers can provide a miniaturized, versatile solution in a relatively low cost setup. The majority of papers in this field described experimental results $[6,7]$ with different designs of fiber optic OT. Even for other applications of OT, there were very few models and systematic studies which could be used to optimize the design and operation of OT. One of the few exceptions was based on the model of Mie for light scattering [6], however, this has been applied only to spherical and near spherical particles [7]. Therefore, it is necessary to develop new tools capable of addressing scatters with different and complex geometry, such as living cells, which will allow designing more effective fiber OT.

This paper is organized as follows: Section 2 describes the theory of calculating the trapping force via the Lorentz force that acts on the dipoles that compose the scattering particle and the simulation method, Section 3 discusses the results, and Section 4 presents the conclusions.

\section{Simulation method}

There has been a strong debate in the literature of how to compute the force produced by an electromagnetic wave on a particle. Two main groups of approaches could be identified. A macroscopic approach based on either the Abraham or Minkowski formulation, considered respectively the kinetic and the canonical momentum in the presence of a scattering object. And also, a microscopic approach based on the Lorentz force which acts on each fundamental constituent of the scattering object $[8,9]$.

The Lorentz theory can be used in two ways. Either it considers that the particle is composed of individual particles or microscopic dipoles [10, 11]. At the microscopic scale, both these approaches lead to the same results, however, at a mesoscopic level the results differ marginally. This work considers only the approach based on the Lorentz force applied on dipoles [9], though the extensions to the other cases can be easily done.

In this case, the Lorentz force density per unit volume acting on the dipoles is

$$
\mathbf{f}^{d}=(\mathbf{P} \cdot \nabla) \mathbf{E}+\frac{\partial}{\partial t}(\mathbf{P} \times \mathbf{B})
$$

where $\mathbf{P}$ is the microscopic polarization, $\mathbf{E}$ is the microscopic electric field, and $\mathbf{B}$ is the microscopic magnetic field.

The microscopic Maxwell equations in the absence of currents and charges are

$$
\begin{gathered}
\nabla \cdot \mathbf{E}=\frac{\rho}{\varepsilon_{0}}, \nabla \cdot \mathbf{B}=0, \\
\nabla \times \mathbf{E}=-\partial_{t} \mathbf{B} \text { and } c^{2} \nabla \times \mathbf{B}=\partial_{t} \mathbf{E}
\end{gathered}
$$

where $c$ is the speed of light in the vacuum.

Combining these equations and (1) yields [12]

$$
f_{i}^{d}=P_{j} \nabla_{i} E_{j}+\frac{\partial}{\partial t}(P \times B)_{i}
$$

where the indexes denote the spatial components of the fields.

When the field is stationary, the second term in (2) is null. Introducing the definition of the macroscopic polarization field

$$
\mathbf{P}=\frac{1}{2} \varepsilon_{0}(\varepsilon-1) \mathbf{E}
$$

into (2) allows to compute

$$
\mathbf{f}^{d}=\frac{1}{2} \varepsilon_{0}(\varepsilon-1) \nabla \mathbf{E}^{2}
$$

where $\varepsilon$ is the relative permittivity of the scattering particle, and $\varepsilon_{0}$ is the vacuum permittivity.

For monochromatic radiation, it is useful to rewrite (4) in terms of the complex macroscopic electric field, resulting in 


$$
\mathbf{f}^{d}=\frac{1}{4} \varepsilon_{0}(\varepsilon-1) \nabla|\mathbf{E}|^{2} .
$$

Equation (5) expresses the force density per unit volume at each point of the particle in terms of the stationary electric field used to trap it. Integrating (5) over the entire volume of the particle gives the net force acting on it.

For low intensity light beams, it is necessary to take into account the reflections of light at the surface of the particle, which was done using the MEEP simulation software. MEEP is an open source program, developed at MIT, which uses the FDTD method [13]. This is widely used to simulate electromagnetic wave propagation. This algorithm is based on the Yee grid discretization to stagger the electric and magnetic field in time and space. A grid with a perfectly matched layer (PML) was implemented to minimize the effects of reflections at the boundary.

The computational grid had 12 per 4 units, with a resolution of $16^{-1}$ and a PML thickness of 0.5 units. The simulation considered a 2D system composed of the long waveguide with a circular tip and a circular particle, as seen in Fig. 1. The surrounding medium had a relative dielectric constant of 1 , and both the particle and the waveguide had a dielectric constant of 2.88. A continuous wave point source with $z$-polarization was located in the middle of the waveguide.

\section{Results}

Figure 2 presents the stationary amplitude distribution of the electric field obtained from the simulations for different positions of the dielectric particles. When the particle is placed exactly in front of the beam, it produces a decrease in the electric field amplitude on the right side of the particle (identifiable in the figure) which results in a momentum transfer from the beam to the particle, producing a force along the optical axis. However, if the particle is placed off the axes, the incident beam is deflected on the scatter, and it produces a kick on the particle in the opposite direction. Both cases exemplify the mechanism of momentum transfer from the beam to the particle, indicating the model validity.

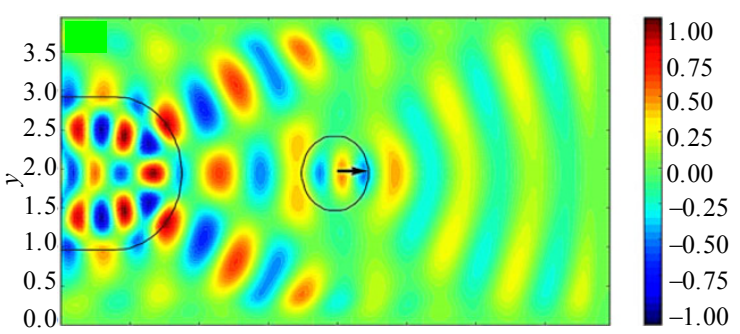

(a)

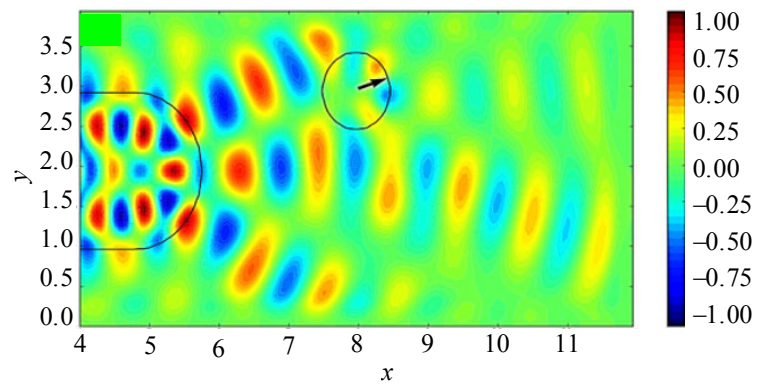

(b)

Fig. 2 Representation of the electric field and net forces acting on the particle: in (a), the particle is placed at the optical axis at $x=8$, while in (b), it is placed off the axes at $y=3.0$ and $x=8.0$ (the spatial units are in micrometers).

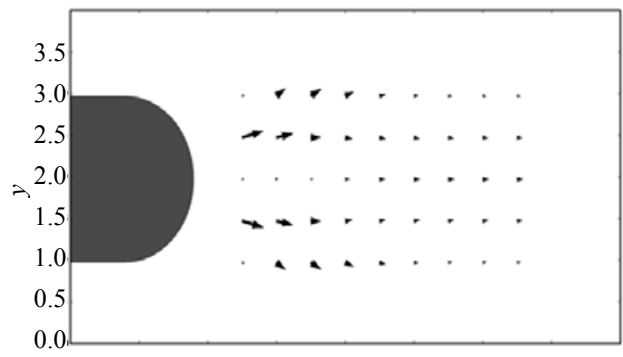

(a)

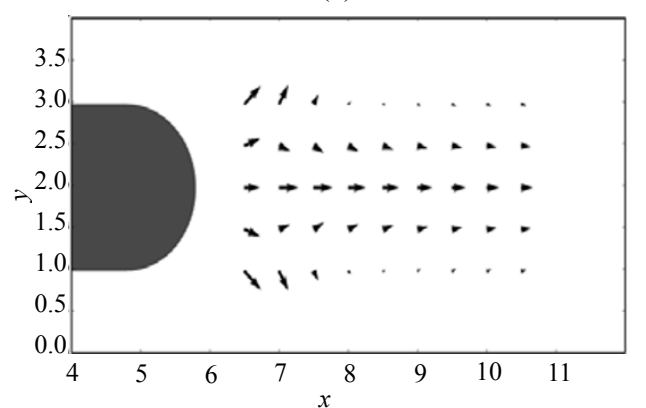

(b)

Fig. 3 Field of forces acting on the beam for particle radii respectively of 0.25 micrometers (a) and 0.5 micrometers (b) (the calculations were done using the normalized field intensity, the amplitude of the force is in arbitrary units and will scale linearly with the power of the light source, and the spatial units are in micrometers). 
Figure 3 represents the field of forces acting on the particle as it moves in front of the beam. Each arrow represents the force acting on the particle if it is placed in that point of space. It can be noticed that, near optical axis of the beam, the particles are attracted to the optical beam axis trapping them while, far from the axis, they are pushed forward. The structure of the field of forces depends on the size of the particle which determines the respective scattering cross sections as well as the profiles of the beam that exits the waveguide.

\section{Conclusions}

This paper presents a method of calculating the forces produced on a dielectric particle by an electromagnetic wave. This method is based on a combination of an FDTD solver (implemented in MEEP) of the electromagnetic field and a microscopic model for the Lorentz force acting on each dipole which constitutes the dielectric particle. Using MEEP takes into account the changes in the field produced by the scattering of light through the particle, thus improving the results relative to previous studies [7]. The preliminary results indicated the suitability of this method to be used in the computation of the force for arbitrary shapes of particles becoming a powerful tool and to optimize the design of new fiber optic tweezers.

Open Access This article is distributed under the terms of the Creative Commons Attribution License which permits any use, distribution, and reproduction in any medium, provided the original author(s) and source are credited.

\section{References}

[1] S. P. Gross. "Application of optical traps in vivo," Methods in Enzymology, vol. 361, pp.162-174, 2003.
[2] W. Hellmich, C. Pelargus, K. Leffhalm, A. Ros, and D. Anselmetti, "Single cell manipulation, analytics, and label-free protein detection in microfluidic devices for systems nanobiology," Electrophoresis, vol. 26, no. 19, pp. 3689-3696, 2005.

[3] G. T. Roman, Y. Chen, P. Viberg, A. H. Culbertson, and C. T. Culbertson, "Single-cell manipulation and analysis using microfluidic devices," Analytical and Bioanalytical Chemistry, vol. 387, no. 1, pp. 9-12, 2007.

[4] D. Wang and S. Bodovitz, "Single cell analysis: the new frontier in 'omics'," Trends in Biotechnology, vol. 28, no. 6, pp. 281-290, 2010.

[5] K. C. Neuman and S. M. Block. "Optical trapping," Review Scientific Instruments, vol. 75, no. 9, pp. 2787-2809, 2004.

[6] P. Minzioni, F. Bragheri, C. Liberale, E. Fabrizio, and I. Cristiani, "A novel approach of fiber-optic tweezers: numerical analysis of the trapping efficiency," IEEE Journal of Selected Topics in Quantum Electronics, vol. 14, no. 1, pp. 151-157, 2008.

[7] R. Taylor and C. Hnatovsky, "Particle trapping in 3-D using a single fiber probe with an annular light distribution," Optics Express, vol. 11, no. 21, pp. 2775-2782, 2003.

[8] Y. Liu and M. Yu, "Investigation of inclined dual-fiber optical tweezers for 3D manipulation and force sensing," Optics Express, vol. 17, no. 16, pp. 13624-13638, 2009.

[9] S. M. Barnett, "Resolution of the Abraham-Minkowski dilemma," Physics Review Letters, vol. 104, no. 7, pp. 070401-1-070401-4, 2010.

[10] S. M. Barnett and R. Loudon, "On the electromagnetic force on a dielectric medium," Journal of Physics B: Atomic, Molecular Optical Physics, vol. 39, no. 15, pp. S671-S684, 2006.

[11] R. Loundon, "Theory of the forces exerted by Laguerre-Gaussian light beams on dielectrics," Physical Review A, vol. 68, no. 1, pp. 013806-1-013806-15, 2003.

[12] R. Loundon, S. M. Barnett, and C. Baxter, "Radiation pressure and momentum transfer in dielectrics: the photon drag effect," Physical Review $A$, vol. 71, no. 6, pp. 063802-1-063802-11, 2003.

[13] A. F. Oskooi, D. Roundy, M. Ibanescu, P. Bermel, J. D. Joannopoulos, and S. G. Johnson, "MEEP: a flexible free-software package for electromagnetic simulations by the FDTD method," Computer Physics Communications, vol. 181, no. 3, pp. 687-702, 2010 